\title{
Spatial Distribution of Voids in Insulating Concrete Analyzed by Micro-CT Images and Probability Functions
}

\author{
Sang-Yeop Chung, ${ }^{1,2}$ Tong-Seok Han,, and Yong-Woo Kim ${ }^{1}$ \\ ${ }^{1}$ Department of Civil and Environmental Engineering, Yonsei University, Yonsei-ro 50, Seodaemun-gu, \\ Seoul 120-749, Republic of Korea \\ ${ }^{2}$ Department of Civil Engineering, Technical University of Berlin, Gustav-Meyer-Allee 25, 13355 Berlin, Germany
}

Correspondence should be addressed to Tong-Seok Han; tshan@yonsei.ac.kr

Received 2 February 2015; Revised 2 April 2015; Accepted 13 April 2015

Academic Editor: Peter Majewski

\begin{abstract}
Copyright (C) 2015 Sang-Yeop Chung et al. This is an open access article distributed under the Creative Commons Attribution License, which permits unrestricted use, distribution, and reproduction in any medium, provided the original work is properly cited.

Insulating concrete is a multiphase material designed for reduced thermal conductivity, and the void distribution in concrete strongly affects its physical properties such as mechanical response and heat conduction. Therefore, it is essential to develop a method for identifying the spatial distribution of voids. To examine the voids of insulating concrete specimens, micro-CT (computed tomography) images can be effectively used. The micro-CT images are binarized to visualize the void distribution and stacked to generate 3D specimen images. From the obtained images, the spatial distribution of the voids and the microscopic constituents inside the insulating concrete specimens can be identified. The void distribution in the material can be characterized using low-order probability functions such as two-point correlation, lineal-path, and two-point cluster functions. It is confirmed that micro-CT images and low-order probability functions are effective in describing the relative degree of void clustering and void connectivity in insulating concrete.
\end{abstract}

\section{Introduction}

The phase distribution of multiphase materials strongly affects their properties [1-3]. The properties of concrete, a random heterogeneous multiphase material that is widely used in infrastructure, are affected by the spatial distribution of each constituent, namely, cement, sand, and aggregate. For example, the strength $[4,5]$ and percolation [6] of a concrete specimen are affected by the spatial distribution of voids within it; therefore, it is essential to investigate the spatial distribution of the constituents of the specimen to clarify its properties.

Insulating concrete is a type of concrete that is designed to have reduced thermal conductivity. Insulating concrete, when cast, contains numerous voids owing to the use of airentraining admixtures or lightweight aggregates. These voids are critical in reducing heat conduction. When the void ratio of the material is increased, certain properties of concrete behave contrarily; with a higher void ratio of the specimen, the insulation is increased, but the strength is decreased. To overcome this drawback, the introduction of anisotropic voids is a promising approach; several studies have examined this topic [5-7]. Therefore, proper methodology to examine the spatial distribution of voids in concrete specimen is necessary for investigating anisotropy of the void distribution in insulating concrete.

Various experimental techniques such as scanning electron microscopy (SEM), optical microscopy (OM), and mercury intrusion porosimetry (MIP) have been used for identifying the inner structure of the void distribution within insulating concrete specimens. SEM and OM can be used to identify the component distribution of cement paste; however, these methods can only provide information obtainable from cross-sectional images. Additional preparation processes, such as polishing and drying, that may damage the specimen are required to obtain more information. MIP is considered effective for investigating the spatial distribution of voids $[8,9]$; however, it cannot describe the overall void 
TABLE 1: Mixing data for insulating concrete.

\begin{tabular}{lcccccc}
\hline W/C ratio (\%) & Cement & Unit quantity of water & Fine aggregate & Glass bead & Total & Note \\
\hline 54.7 & 320 & 175 & 827 & 45 & 1373 & Unit weight $\left(\mathrm{kg} / \mathrm{m}^{3}\right)$ \\
\hline
\end{tabular}

distribution within the material, and the specimen may be damaged during drying before MIP testing and pressure loading [10].

In this study, computed tomography (CT), a nondestructive and noninvasive method, is used to investigate the spatial distribution of the constituents of insulating concrete. In this method, a series of cross-sectional images of insulating concrete are generated using X-rays without damaging the specimen. These images are expressed by a pixel, which serves as a unit for building two-dimensional (2D) images. A CT imaging process that converts images from an 8-bit image to a binary image is used to examine the specific constituents of insulating concrete. Subsequently, three-dimensional (3D) images of the insulating concrete can be generated by stacking the $2 \mathrm{D}$ images.

Numerous studies have reported on the use of CT imaging for investigating material characteristics. Lu et al. [11] used $\mu \mathrm{CT}$ to examine the pore connectivity and its correlation with chloride migration in order to evaluate the permeability of concrete. Masad et al. [12] used CT to evaluate the permeability and anisotropy of asphalt by simulating the microstructure. Gallucci et al. [13] used $\mu \mathrm{CT}$ to investigate the degree of the pore network in concrete. CT images have also been used for studying other properties of concrete materials. Burlion et al. [14] used CT images to analyze the microstructure of cementitious materials during leaching. Chotard et al. [15] used CT to evaluate the early stage of the hydration of calcium aluminate cement. Helfen et al. [16] used CT to examine the evolution of the cement microstructure during hydration. Chung et al. [17] used CT images to evaluate the correlation between the anisotropy of the void distribution and the stiffness of lightweight aggregates. Rougelot et al. [18] used CT images to examine microcracking in cement composites. Zhang et al. [10] used $\mu \mathrm{CT}$ to investigate mass diffusivity in cement paste. These studies have adopted CT imaging methods to investigate the characteristics and properties of concrete materials.

In addition to $\mathrm{CT}$ imaging, probability-based methods are employed to describe the void distribution in insulating concrete. Here, the void distribution of insulating concrete was characterized using low-order probability functions such as two-point correlation $[3,19]$, lineal-path $[1,20]$, and twopoint cluster $[21,22]$. The degree of phase clustering can be identified using a two-point correlation function $[1,3]$. The lineal-path function can be used to obtain information about the phase connectivity along a specific direction. The twopoint cluster function is effective for describing the continuity of phase-clustering-related material characteristics such as percolation and diffusivity $[1,21]$.

In this study, the spatial distribution of voids in concrete specimens with different constituents is investigated. In insulating concrete, hollow glass beads $(3 \mathrm{M})$ with diameters of the order of a few micrometers are added to increase
TABLE 2: Material properties of glass bead.

\begin{tabular}{lc}
\hline Properties & Specification \\
\hline Composition & Soda-lime borosilicate glass \\
Color & White \\
Density & $125 \mathrm{~kg} / \mathrm{m}^{3}$ \\
Crushed strength & $1.72 \mathrm{MPa}$ \\
Softening temperature & $600^{\circ} \mathrm{C}$ \\
Diameter & $10 \sim 65 \mu \mathrm{m}$ \\
Bulk thermal conductivity & $0.044 \mathrm{~W} / \mathrm{mk}$ \\
\hline
\end{tabular}

the insulating effect. Therefore, a $\mu \mathrm{CT}$ image expressed by a micrometer order pixel size is required to identify the voids induced by the glass beads in the insulating concrete specimen. To obtain high-resolution CT images, the highenergy micro-CT facility in the 6D X-ray imaging beamline at Pohang Accelerator Laboratory (PAL, Korea) is used. The spatial distribution of glass beads as well as voids in the insulating concrete is examined using $\mu \mathrm{CT}$ images and low-order probability functions. Using low-order probability functions, the characteristics of the void distribution can be described using only a small amount of data. Anisotropy of the void distribution inside the specimen is also examined using those methods. In addition, the difference between the void distribution of concrete specimens with and without glass beads is compared to investigate the effect of the glass beads on the cementitious material.

\section{Insulating Concrete Specimen and $\mu$ CT Imaging}

2.1. Insulating Concrete Specimens. Insulating concrete is widely used as an exterior and interior structural material to improve energy efficiency by reducing heat loss through its insulating effect [23-25]. Studies have extensively focused on using lightweight aggregates to produce numerous voids in concrete and thus to improve the insulation [26, 27]. In this study, hollow glass beads (3M) were added to insulating concrete to enhance its insulating effect. The insulating concrete specimen is prepared using the mix design shown in Table 1. The material properties of the glass bead used are listed in Table 2. These beads have diameters of 10-65 $\mu \mathrm{m}$, and they are used to produce a spherical void within the concrete. The beads are expected to be more effective for insulation than lightweight aggregates because they can ensure regularly dispersed voids within the concrete.

An insulating concrete specimen composed Type I Portland cement, fine aggregates, and glass beads. The specimen is produced using a plastic tube with a length and diameter of $70 \mathrm{~mm}$ and $4 \mathrm{~mm}$, respectively, as shown in Figure 1. The specimen volume is the maximum size that can be analyzed 

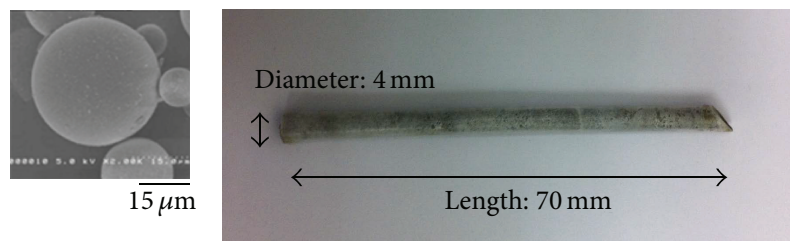

FIGURE 1: Shape of glass bead (left) and insulating concrete specimen (right).

effectively using the X-ray micro-CT device at PAL. The concrete specimen is cured under standard conditions $\left(25^{\circ} \mathrm{C}\right)$ for 28 days. In this study, the fine aggregates used are washed ones having a fineness modulus (FM) of 2.64; aggregates smaller than $1.2 \mathrm{~mm}$ in size are only used in consideration of the specimen size.

2.2. CT Imaging for Constituents Analysis. It is difficult to investigate the constituent distribution of concrete without damaging the specimen. In this study, CT imaging is used to examine the spatial distribution of constituents as well as voids within insulating concrete. Cross-sectional images of insulating concrete are obtained using the micro-CT facility at Pohang Accelerator Laboratory (PAL) in Korea. It is essential to use the synchrotron micro-CT device because the images from a general CT device (e.g., [6]) cannot visualize glass beads having diameters of the order of a few micrometers. At PAL, an X-ray radiograph and a phase difference image with micrometer resolution can be obtained using the maximum $55 \mathrm{keV}$ X-ray source. Figure 2(a) shows the insulating concrete specimen setup and Figure 2(b) the synchrotron micro CT equipment. A cross-sectional CT image of the insulating concrete specimen is generated when $\mathrm{X}$-rays are injected from the device shown in Figure 2(c) and transmitted to the specimen.

Figure 3 shows sample 8-bit micro-CT cross-sectional images of the insulating concrete specimen, in which a pixel is represented by a value ranging from 0 to 255 (total of 256 values) depending on the relative density, where 0 is black and 255 is white in gray scale. A pixel value of 0 indicates that the relative density of the material for the pixel is minimum. Each micro-CT image is composed of $1633 \times 1633(x \times y)$ pixels with $3.4 \mu \mathrm{m}$ pixel size, and 1,500 images are obtained along the length direction over a $5.1 \mathrm{~mm}$ range. In the figure, the thin circular border is a plastic tube that encloses the insulating concrete specimen, and the blurred region outside the border is noise which is a byproduct of image reconstruction.

Assuming that cement is not completely hydrated and a unit quantity of water is fully evaporated, the constituents in the plastic tube are voids, glass beads $\left(\rho=0.125 \mathrm{~kg} / \mathrm{m}^{3}\right)$, mortar $\left(\rho=1.43 \mathrm{~kg} / \mathrm{m}^{3}\right)$, and the fine aggregates $(\rho=$ $2.58 \mathrm{~kg} / \mathrm{m}^{3}$ ) remaining after the hydration reaction, where $\rho$ is the material density. Therefore, the darkest region in Figure 3 can be considered as glass beads or voids and the brightest region as fine aggregate with relatively large density. The gray region observed across most of the specimen is considered to be mortar. As shown in Figure 3, the spatial distribution of the constituents in the insulating concrete specimen can be determined effectively and nondestructively using $\mu \mathrm{CT}$ images obtained from the synchrotron facility. To quantify the spatial distribution, probabilistic functions can be used.

\section{Low-Order Probability Functions for Material Characterization}

A suitable method is required for determining the spatial distribution of the constituents of the insulating concrete specimen. In this study, low-order probability functions, originally used to characterize the phase distribution of random heterogeneous materials [1], are used for this purpose.

3.1. Two-Point Correlation Function. The two-point correlation function $\left(P_{i j}(r, \theta, \phi)\right)$ is used to characterize the phase distribution in this research. $P_{i j}(r, \theta, \phi)$ is the probability that any two points are located in the $i$-phase and $j$-phase $[1,3,17,19] . r$ is the distance between two points; $\theta$ is the angle between a test line and the $z$-axis; and $\phi$ is the angle between the projection of a test line on the $x y$-plane and the $x$-axis, as shown in Figure 4.

The two-point correlation function only includes phase information at the end points of the test line; therefore, any additional information between two points is not included in this function. The general limits for the two-point correlation function are

$$
\begin{aligned}
& \lim _{r \rightarrow 0} P_{i i}(r, \theta, \phi)= f_{i}, \\
& \lim _{r \rightarrow 0} P_{i j}(r, \theta, \phi)= 0, \\
&(i \neq j) . \\
& \lim _{r \rightarrow \infty} P_{i i}(r, \theta, \phi)= {\left[f_{i}\right]^{2}, } \\
& \lim _{r \rightarrow \infty} P_{i j}(r, \theta, \phi)=\left[f_{i}\right]\left[f_{j}\right], \\
& \quad(i \neq j),
\end{aligned}
$$

where $f_{i}$ is the volume fraction of phase $i$. In (1), when the distance between two points approaches zero, the probability of finding the same phase at both points converges to the phase volume fraction; otherwise, the probability of finding different phases at a point becomes zero. Equation (2) indicates that $P_{i j}(r)$ converges to the product of the phase volume fraction as $r$ increases. Gokhale et al. [19] suggested a simple form of the two-point correlation function based on statistical information [28]

$$
\begin{array}{r}
P_{i j}(r, \theta, \phi)=f_{i} f_{j}\left[1-\exp \left\{-\left(\frac{\left[P_{L}(\theta, \phi)\right]_{i j}}{\left(2 f_{i} f_{j}\right)}\right) r\right\}\right], \\
(i \neq j) .
\end{array}
$$

In (3), $\left[P_{L}(\theta, \phi)_{i j}\right]$ is the number of intersections between a test line and the $i$ - $j$ phase interface per unit test line length. In the case of two-phase materials, the probability that both 


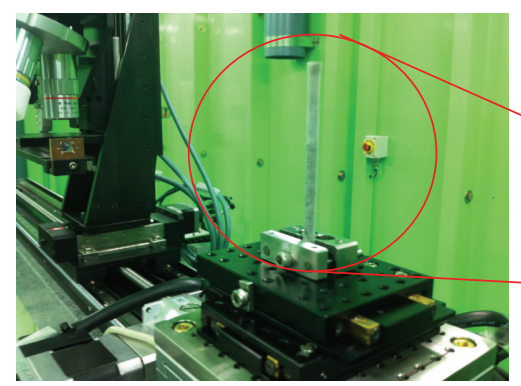

(a)

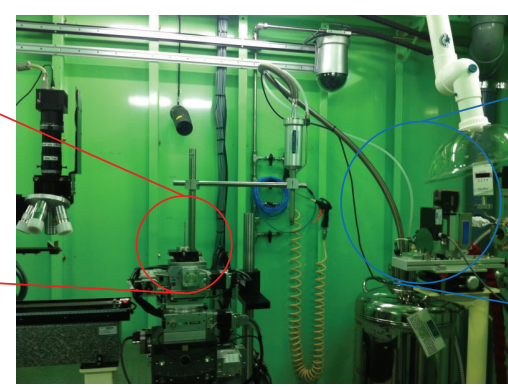

(b)

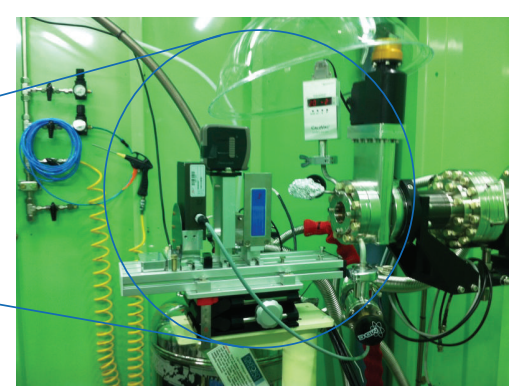

(c)

FIGURE 2: Specimen and CT image equipment using high-energy X-ray (PAL): (a) specimen setting, (b) micro-CT equipment, and (c) X-ray injection part.

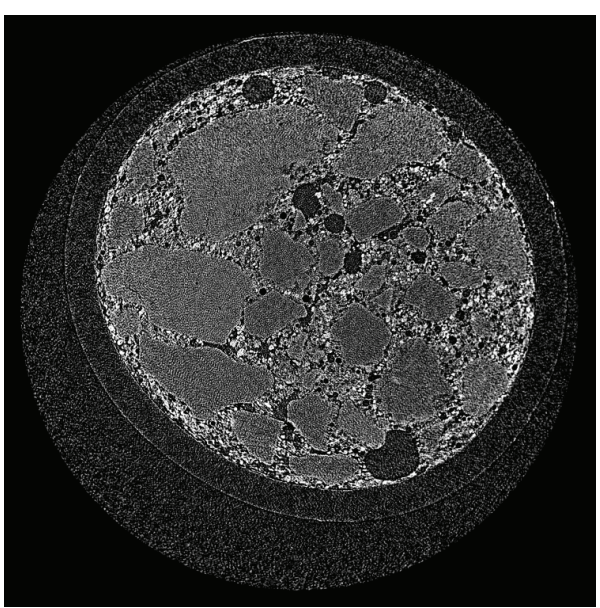

(a)

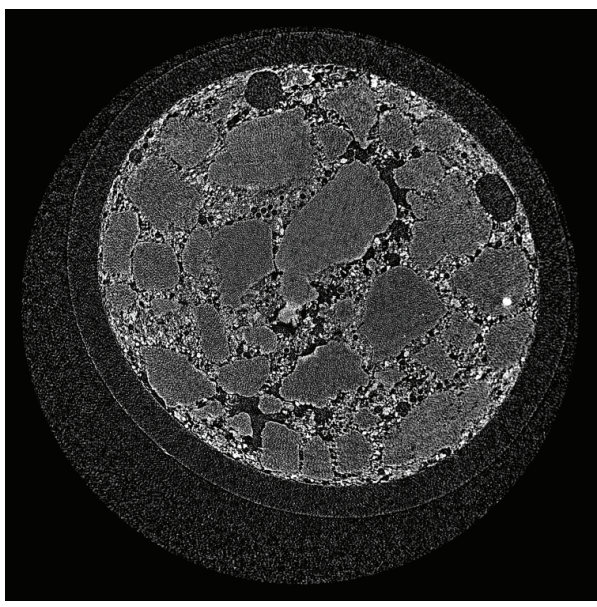

(c)

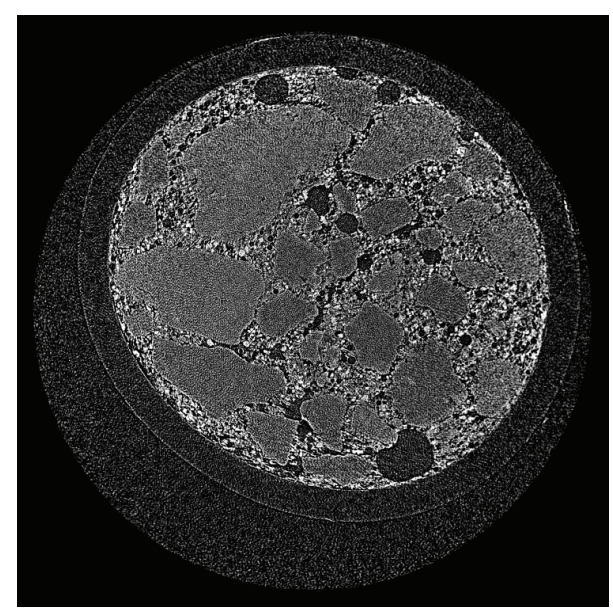

(b)

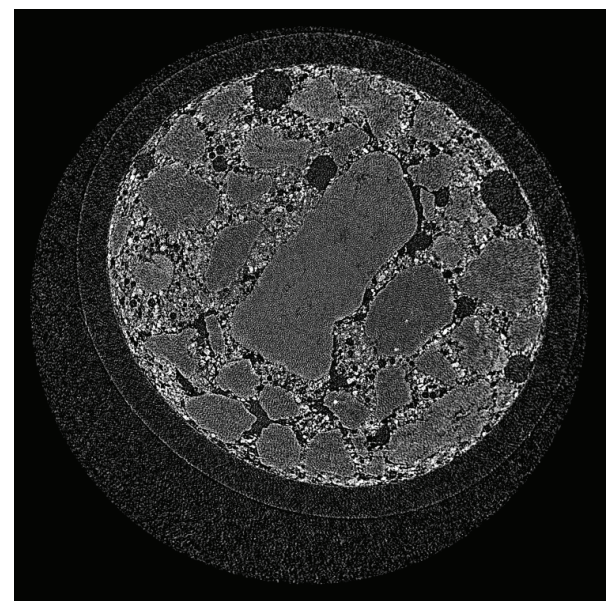

(d)

FIGURE 3: 8-bit micro CT cross-sectional images of insulating concrete (1,500 images are obtained along the length direction: (a) 100th image, (b) 138th image, (c) 400th image, and (d) 500th image).

phases are located in the same $(i)$ phase, $P_{i i}$, can be obtained from $P_{i j}$ (3) and

$$
P_{i i}(r, \theta, \phi)+P_{i j}(r, \theta, \phi)=f_{i}
$$

3.2. Lineal-Path Function. To further investigate the constituent distribution, a lineal-path function is used to compensate for the two-point correlation function. The linealpath function, $L_{i}(r, \theta, \phi)$, is the probability that a randomly 


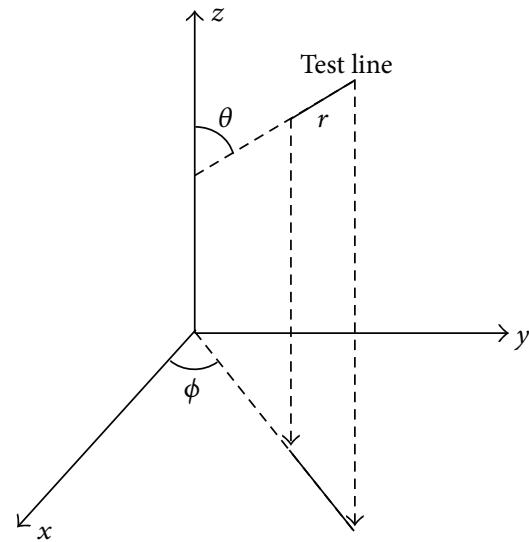

FIGURE 4: Specification of orientation in 3D space.

placed line of length $r$ is entirely in a phase $i[6,29,30]$. Using the lineal-path function, the continuous connectivity of the same phases for a specific direction can be evaluated. The general limits for the lineal-path function are

$$
\begin{aligned}
& \lim _{r \rightarrow 0} L_{i}(r, \theta, \phi)=f_{i}, \\
& \lim _{r \rightarrow \infty} L_{i}(r, \theta, \phi)=0 .
\end{aligned}
$$

In this study, Coker and Torquato's method [20] is adopted to obtain the lineal-path function for the insulating concrete specimen. To calculate the lineal-path function, a random point inside the specimen is selected, and the phase information for the specific direction is analyzed by increasing the length of the test line. This process is repeated until a test line reaches a different phase from that of the starting point. From the lineal-path function, the connectivity of the specific constituent can be evaluated because it considers the entire line segment between two end points of the test line.

3.3. Two-Point Cluster Function. Another form of the probability function - the two-point cluster function-is used to examine the connectivity of both the phase distribution and the phase clusters. The two-point cluster function, $C_{i}(r, \theta, \phi)$, is defined as the probability of finding any two points in the same cluster of phase $i[1,21]$. Using the two-point cluster function, the connected cluster of a phase can be identified even when the phase connectivity along the specific direction is interrupted by another phase. The general limits for the two-point cluster function are

$$
\begin{aligned}
& \lim _{r \rightarrow 0} C_{i}(r, \theta, \phi)=f_{i}, \\
& \lim _{r \rightarrow \infty} C_{i}(r, \theta, \phi)=0 .
\end{aligned}
$$

To calculate the two-point cluster function, Lee and Torquato's method [22] is used. In this method, two points in the material are arbitrarily selected, and it is checked whether these two points are located in the same phase and cluster. Then, the distance between these two points is calculated, and this process is repeated along the specific direction to obtain the two-point cluster function with the change of $r$. The two-point cluster function can be used to obtain detailed information on the phase distribution because it provides information about the connectivity for both the phase and the cluster. Thus, the two-point cluster function can be used for a material that needs to secure the channel pass, such as insulating and porous concrete.

\section{Analysis of Constituents of Insulating Concrete Specimen}

In this study, we determine the void distribution within insulating concrete using $\mu \mathrm{CT}$ images and analyze the spatial distribution of voids quantitatively using probability functions. The void distribution of insulating concrete strongly affects material properties such as thermal conductivity and strength. Using $\mu \mathrm{CT}$ images obtained from a synchrotron facility, voids within the specimen as well as visible ones on the surface are described. In addition, the spatial distribution of voids is statistically characterized using low-probability functions.

4.1. CT Imaging of Insulating Concrete Specimen. The spatial distribution of the constituents of insulating concrete can be roughly identified from Figure 3. For example, regions 1-5 in Figure 5(a) are samples of circular voids. In this image, a circular void having a diameter of at most 20 pixels can be considered a glass bead because the maximum diameter of a glass bead is $65 \mu \mathrm{m}$ (20 pixels based on a pixel size of $3.4 \mu \mathrm{m}$ ). Therefore, among the voids in Figure 5(a), regions 4 and 5 can be considered glass beads. Figure 5(b) shows a 3D image of a glass bead as obtained subsequently by stacking 8 -bit crosssectional images around region 5 in Figure 5(a); because a smooth sphere is identified, region 5 can be considered a candidate for a glass bead. As shown in Figure 5, the constituents of insulating concrete can be investigated using $\mu \mathrm{CT}$ images.

However, with the 8-bit $\mu \mathrm{CT}$ image, it is difficult to detect minute components such as sparsely dispersed voids inside the specimen. In addition, the objective of the use of glass beads is to secure the void inside the specimen; the void phase, including the voids induced by glass beads, is the primary element of interest in this study. To describe the void distribution inside the insulating concrete effectively, a binary image is generated from the 8-bit cross-sectional image, as shown in Figure 6. To analyze the void distribution inside the insulating concrete specimen, the region of interest (Figure 6(b)) is extracted from the original $\mu \mathrm{CT}$ image shown in Figure 6(a). In the 8-bit image in Figure 6(b), a set of pixels smaller than the threshold value is considered as voids. Here, a threshold value is selected using the Otsu method [31] from the gray-level histogram of the image, and the median filter is also used for noise reduction. By using an imaging process to separate voids and solids, a binary image is generated from the 8-bit cross-sectional image, as shown in Figure 6(c). For binary imaging, the image processing toolbox in MATLAB [32] is used. The binary image in Figure 6(c) is composed of $700 \times 700(x \times y)$ pixels with a pixel size of $3.4 \mu \mathrm{m}$. In 


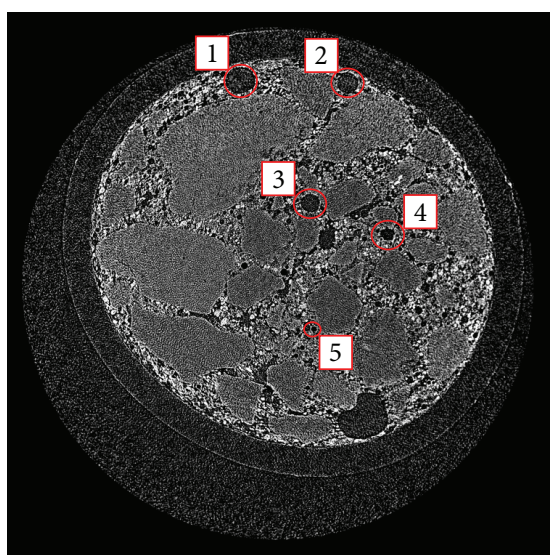

(a)

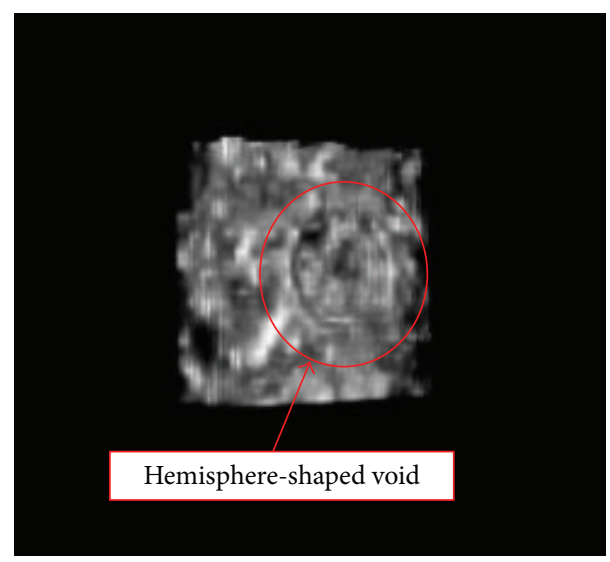

(b)

FIGURE 5: Region of voids and glass beads inside the insulating concrete specimen: (a) inner voids of the specimen (voids 1, 2, and 3: voids in cement, voids 4 and 5: candidates for glass bead) and (b) sample of 3D void image by glass bead.

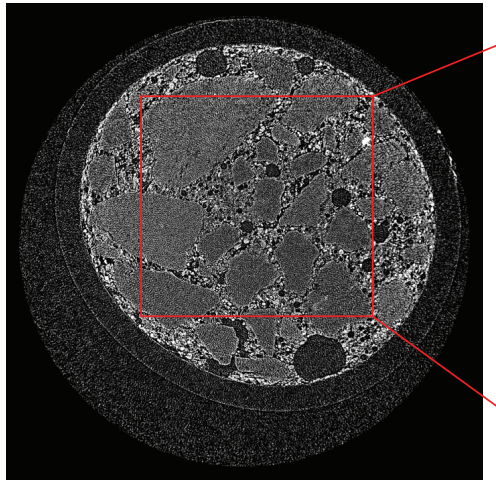

(a)
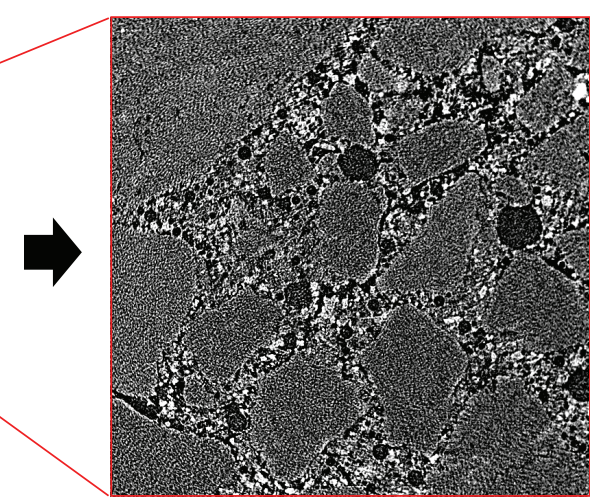

(b)

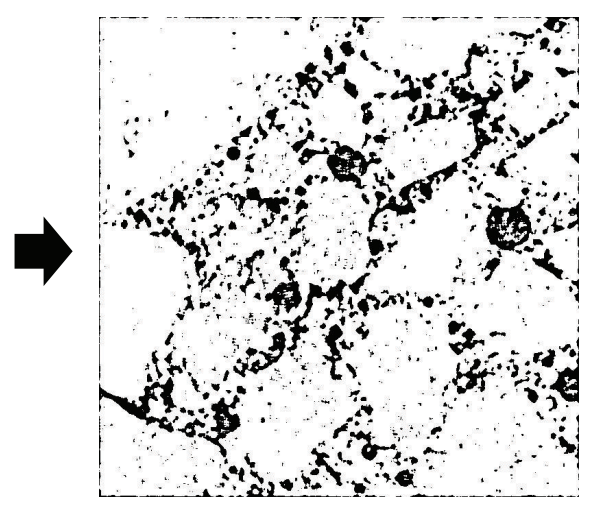

(c)

FIGURE 6: $\mu \mathrm{CT}$ imaging to generate a binary image: (a) original 8-bit CT image, (b) squared cross-sectional image, and (c) binary image (white: solid region, black: voids within specimen).

this figure, the white region is solid, and the black region is a void within the insulating concrete specimen. To describe the void distribution of the insulating concrete specimen in $3 \mathrm{D}$, a series of binary images are subsequently stacked. The 3D image in Figure 7(a) is generated by stacking 700 binary images along the $z$-direction, and it is composed of $700 \times 700$ $\times 700(x \times y \times z)$ voxels. The concrete model in Figure 7 is the region of interest (ROI) selected from the insulating concrete specimen. Figure 7(b) shows the void image of the specimen. From this image, both large void clusters and minute sparsely dispersed voids inside the specimen can effectively be detected; it can be denoted that the image resolution used in this study is sufficient to describe capillary pores including the void induced by glass beads. Using the image in Figure 7, the void ratio as well as the spatial distribution of voids inside the insulating concrete specimen can be identified. To quantify the void distribution in the specimen, the probabilistic methods discussed in Section 3 can be used.

4.2. Quantitative Description of Void Distribution Using Probability Functions. In this study, to quantitatively describe the spatial distribution of voids, three low-order probability functions-two-point correlation $\left(P_{v v}\right)$, lineal-path $\left(L_{v}\right)$, and two-point cluster $\left(C_{v}\right)$ functions-are used. Here, the subscript $v$ indicates the void phase. Figure 8 shows probability functions for the ROI of the insulating concrete specimen for the $x, y$, and $z$ directions. In each function, $r$ is the distance between two points, and $D$ is the length of the specimen in the axial direction. The two-point correlation function for voids, $P_{v v}(r)$, is shown in Figure $8(\mathrm{a})$, and it is found to be similar for the $x, y$, and $z$ directions. The relative degree of void clustering for different directions can be identified using $P_{v v}(r)$; large $P_{v v}(r)$ values for a specific direction indicates that more voids are clustered in that direction. Therefore, Figure 8(a) indicates that the degree of void clustering in the specimen is almost isotropic or statistically identical for all axial directions.

Figure 8(b) shows $L_{v}(r)$ for the $x, y$, and $z$ directions; it is similar in all three directions, indicating that the straightline void connectivity is isotropic in all three directions, even though a small difference is found in Figure 8(b). Similarly, the two-point cluster function $\left(C_{v}(r)\right)$ in Figure 8(c) shows 


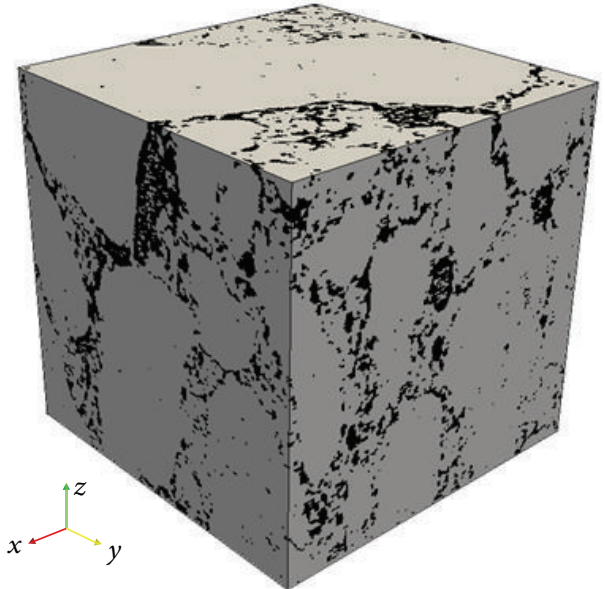

(a)

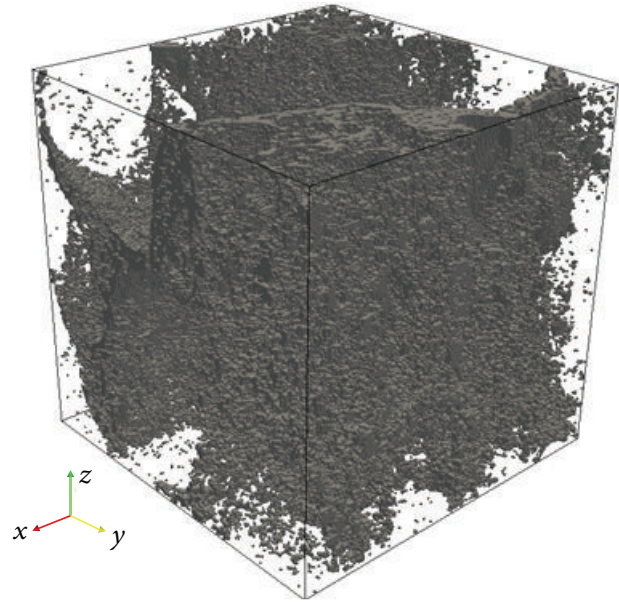

(b)

FIGURE 7: 3D binary image of insulating concrete specimen (void ratio $=10.93 \%$ ): (a) whole binary image and (b) void distribution within specimen.

an isotropic void distribution when $r / D$ is $0-0.2$. However, anisotropic fluctuation of $C_{v}(r)$ is observed when $r / D$ is $0.2-$ 0.6. This indicates the existence of anisotropic continuous void clusters with different sizes in the specimen. These results indicate that the relative size of the void clustering within a specimen can be quantitatively identified using the probability functions; Figure 8 indicates that a specific region of the specimen contains an anisotropic continuous void cluster. Continuous void clusters passing through the specimen that affect material properties, such as permeability, can also be identified using the two-point cluster function. The existence of a void cluster passing through the entire specimen can be identified from the $C_{v}(r)$ value when $r / D$ is 1 ; the function values are not equal to zero when the percolating void clusters exist in the specimen. In addition, the applicability of the specimen as a representative volume element (RVE, the smallest subset of material volume that describes representative properties) can be identified from the probability function; when the specimen is a RVE, each probability function for all directions should be the same because a RVE of concrete is isotropic. From the two-point correlation and lineal-path functions, the concrete sample investigated in this study can be considered as a RVE of the specimen because their values are almost the same for all axial directions. However, the result of the two-point correlation function in Figure 8(c) demonstrates that this sample contains relatively large and anisotropic void clusters passing through the specimen, which confirms that this sample might not be considered as a RVE of the specimen; for more detailed investigation about the applicability of a RVE, the use of a larger sample will be needed.

To evaluate the effect of glass beads on the concrete specimen, CT images of the specimens with/without glass beads are compared. Figures 9(a) and 9(b), respectively, show cross-sectional images of the specimen with and without glass beads. The two cases differ owing to the existence of glass beads in the specimen. The void ratio of the specimen with glass beads (Figure 9(a)) is 10.93\%, and that of the specimen without glass beads (Figure $9(\mathrm{~b})$ ) is $6.84 \%$. As shown in the binary images in Figure 9, the voids distributed between the fine aggregate (white region) are more distinctive in Figure 9(a) than in Figure 9(b). Voids induced by glass beads are found to restrict the chemical reaction between aggregates. Therefore, more voids are dispersed between aggregates in the specimen that contains glass beads, and this phenomenon can be inferred from the CT images in Figure 9.

The results of this study show that the void distribution within insulating concrete can be characterized using $\mu \mathrm{CT}$ imaging and probabilistic functions. In addition, the effect of specific constituents (e.g., glass beads) on the concrete material can also be examined from $\mu \mathrm{CT}$ images.

\section{Conclusions}

This study investigated methods for characterizing the spatial distribution of the constituents of insulating concrete. For insulating concrete, a specimen that contained hollow glass beads to increase the insulating effect was used. In general, glass beads having a diameter of a few micrometers can be utilized to secure regularly distributed voids within the specimen. To visualize the void distribution in the concrete specimen without damaging it, the high-energy micro-CT facility at the PAL synchrotron was used. The synchrotron device was necessary to obtain a high-resolution image because the constituent, for example, glass bead, can only be described in the CT image with micrometer pixel size.

The specific constituents in the insulating concrete specimen were identified using $\mu \mathrm{CT}$ images. In particular, the void distribution within the ROI of the insulating concrete was described using a series of $\mu \mathrm{CT}$ images and CT image processing. The spatial distribution of voids in the specimen could be visualized using the 3D digital insulating concrete image generated by the subsequent stacking of 2D binarized images. To quantify the void distribution of the specimen, 


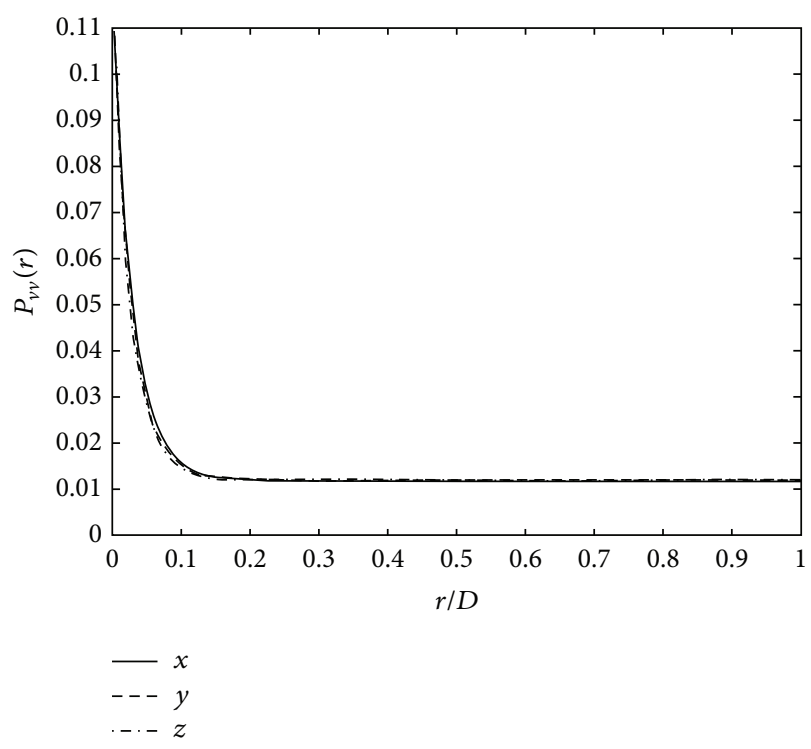

(a)

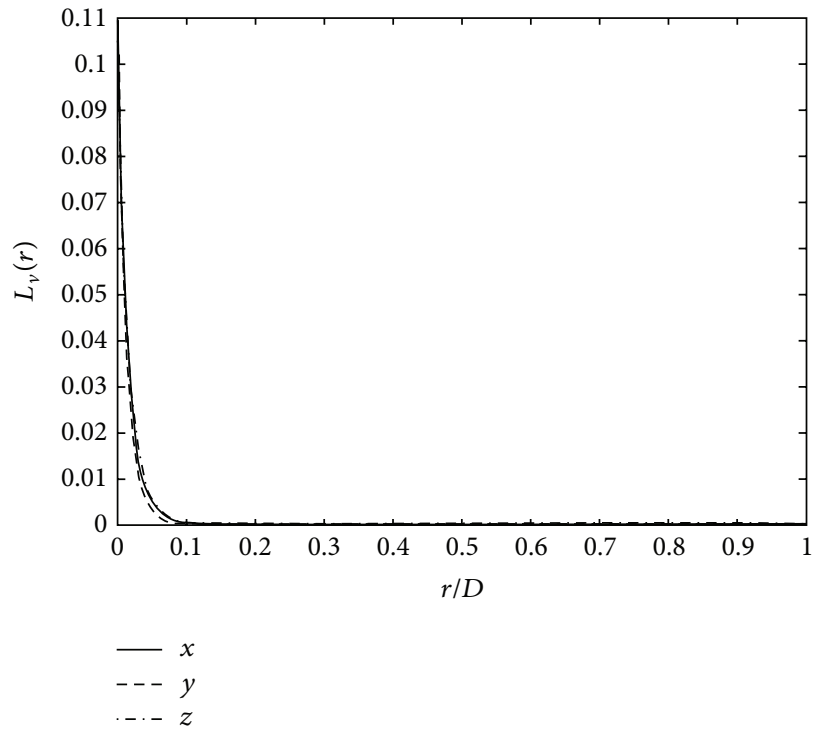

(b)

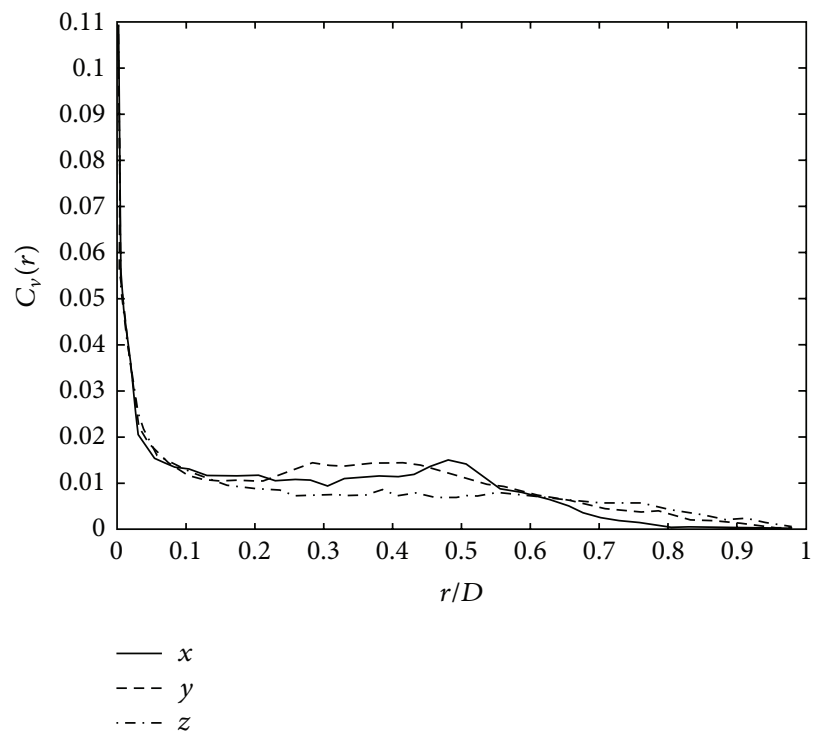

(c)

FIGURE 8: Low-order probability functions for the ROI of the insulating concrete specimen: (a) two-point correlation function, (b) lineal-path function, and (c) two-point cluster function (note: $r$ is the distance between two points, and $D$ is the length of the specimen side).

probabilistic methods such as two-point correlation, linealpath, and two-point cluster functions were adopted. From these low-order probability functions, the degree of void clustering and void connectivity can be characterized. The isotropy of the void distribution in the specimen was also demonstrated from the probability functions.

The results confirm that $\mu \mathrm{CT}$ imaging with a highresolution image and a low-order probability function can be effectively used to examine the spatial distribution of the voids in insulating concrete. Anisotropy of the void distribution was effectively examined by using these methods effectively, and the applicability of the specimen as a RVE was also identified. In particular, the heterogeneity and anisotropy of the void distribution were identified by using these methods, which can be used to design an optimized insulating concrete model with appropriate void size and distribution. Further simulation studies of heat conduction and mechanical behavior for insulating concrete samples might be necessary to evaluate the relationship between void distribution and material properties, such as thermal conductivity and strength of insulating concrete, in detail.

\section{Conflict of Interests}

The authors declare that there is no conflict of interests regarding the publication of this paper. 

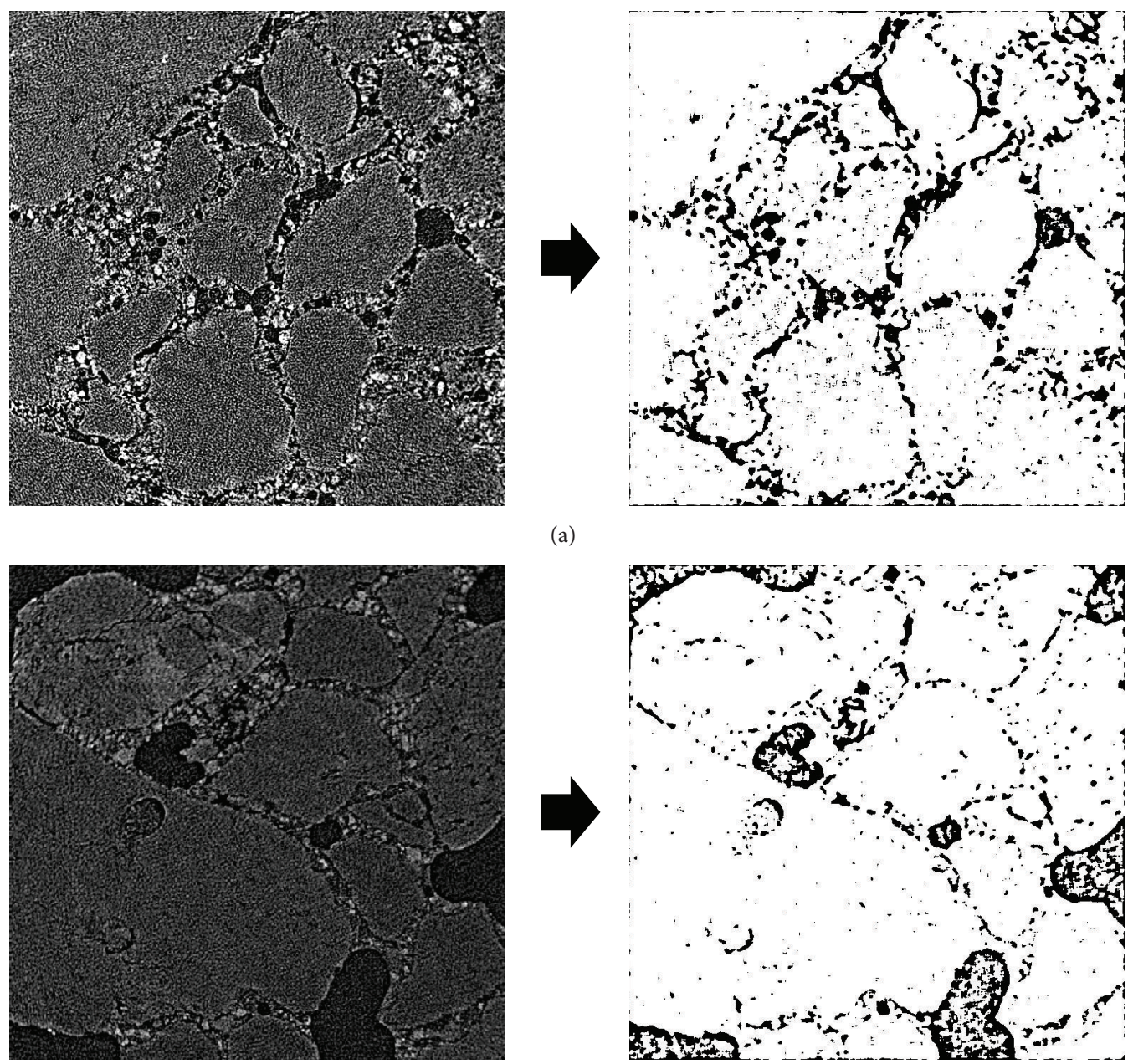

(a)

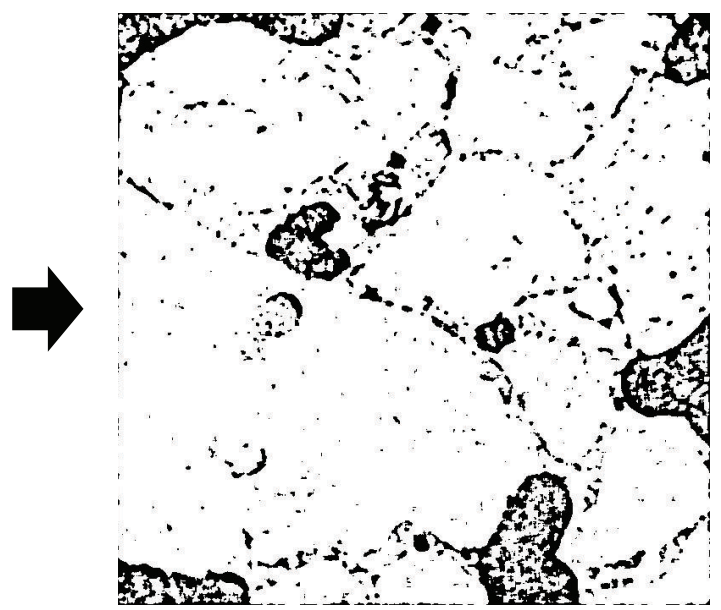

(b)

FIGURE 9: Cross-sectional images of the specimen with/without glass beads (left: 8-bit image, right: binary image (white: solid, black: void)): (a) image of insulating concrete specimen with glass beads, (b) image of concrete specimen without glass beads.

\section{Acknowledgments}

This research was supported by a Korea Research Foundation grant funded by the Korean Government (KRF-2011-0029212 and KRF-2012R1A1A2006629). This work was also supported by the Industrial Strategic Technology Development Program (10041589) funded by the Ministry of Knowledge Economy (MKE, Korea). The authors acknowledge Pohang Accelerator Laboratory (PAL) for beamline use (Grant 2012-1st-6D004). In addition, the authors express their appreciation to Dr. Kwang Soo Youm at GS E\&C Research Institute in the Republic of Korea, who provided insulating concrete specimens and physical property data.

\section{References}

[1] S. Torquato, Random Heterogeneous Materials, Springer, New York, NY, USA, 2002.

[2] T.-S. Han and P. R. Dawson, "Representation of anisotropic phase morphology," Modelling and Simulation in Materials Science and Engineering, vol. 13, no. 2, pp. 203-223, 2005.
[3] S.-Y. Chung and T.-S. Han, "Reconstruction of random twophase polycrystalline solids using low-order probability functions and evaluation of mechanical behavior," Computational Materials Science, vol. 49, no. 4, pp. 705-719, 2010.

[4] D. N. Winslow, M. D. Cohen, D. P. Bentz, K. A. Snyder, and E. J. Garboczi, "Percolation and pore structure in mortars and concrete," Cement and Concrete Research, vol. 24, no. 1, pp. 2537, 1994.

[5] R. A. Dorey, J. A. Yeomans, and P. A. Smith, "Effect of pore clustering on the mechanical properties of ceramics," Journal of the European Ceramic Society, vol. 22, no. 4, pp. 403-409, 2002.

[6] S.-Y. Chung and T.-S. Han, "Correlation between low-order probability distribution functions and percolation of porous concrete," Magazine of Concrete Research, vol. 65, no. 7, pp. 448460, 2013.

[7] R. Cabrillac, B. Fiorio, A.-L. Beaucour, H. Dumontet, and S. Ortola, "Experimental study of the mechanical anisotropy of aerated concretes and of the adjustment parameters of the introduced porosity," Construction and Building Materials, vol. 20, no. 5, pp. 286-295, 2006.

[8] S. Diamond, "Mercury porosimetry: an inappropriate method for the measurement of pore size distributions in cement-based 
materials," Cement and Concrete Research, vol. 30, no. 10, pp. 1517-1525, 2000.

[9] R. Kumar and B. Bhattacharjee, "Study on some factors affecting the results in the use of MIP method in concrete research," Cement and Concrete Research, vol. 33, no. 3, pp. 417-424, 2003.

[10] M. Zhang, Y. He, G. Ye, D. A. Lange, and K. V. Breugel, "Computational investigation on mass diffusivity in Portland cement paste based on X-ray computed microtomography $(\mu \mathrm{CT})$ image," Construction and Building Materials, vol. 27, no. 1, pp. 472-481, 2012.

[11] S. Lu, E. N. Landis, and D. T. Keane, "X-ray microtomographic studies of pore structure and permeability in Portland cement concrete," Materials and Structures, vol. 39, no. 6, pp. 611-620, 2006.

[12] E. Masad, A. Al Omari, and H.-C. Chen, "Computations of permeability tensor coefficients and anisotropy of asphalt concrete based on microstructure simulation of fluid flow," Computational Materials Science, vol. 40, no. 4, pp. 449-459, 2007.

[13] E. Gallucci, K. Scrivener, A. Groso, M. Stampanoni, and G. Margaritondo, "3D experimental investigation of the microstructure of cement pastes using synchrotron X-ray microtomography $(\mu \mathrm{CT})$," Cement and Concrete Research, vol. 37, no. 3, pp. 360-368, 2007.

[14] N. Burlion, D. Bernard, and D. Chen, "X-ray microtomography: application to microstructure analysis of a cementitious material during leaching process," Cement and Concrete Research, vol. 36, no. 2, pp. 346-357, 2006.

[15] T. J. Chotard, M. P. Boncoeur-Martel, A. Smith, J. P. Dupuy, and C. Gault, "Application of X-ray computed tomography to characterise the early hydration of calcium aluminate cement," Cement and Concrete Composites, vol. 25, no. 1, pp. 145-152, 2003.

[16] L. Helfen, F. Dehn, P. Mikulik, and T. Baumbach, "Threedimensional imaging of cement microstructure evolution during hydration," Advances in Cement Research, vol. 17, no. 3, pp. 103-111, 2007.

[17] S.-Y. Chung, T.-S. Han, T. S. Yun, and K. S. Youm, "Evaluation of the anisotropy of the void distribution and the stiffness of lightweight aggregates using CT imaging," Construction and Building Materials, vol. 48, pp. 998-1008, 2013.

[18] T. Rougelot, N. Burlion, D. Bernard, and F. Skoczylas, "About microcracking due to leaching in cementitious composites: $\mathrm{X}$ ray microtomography description and numerical approach," Cement and Concrete Research, vol. 40, no. 2, pp. 271-283, 2010.

[19] A. M. Gokhale, A. Tewari, and H. Garmestani, "Constraints on microstructural two-point correlation functions," Scripta Materialia, vol. 53, no. 8, pp. 989-993, 2005.

[20] D. A. Coker and S. Torquato, "Extraction of morphological quantities from a digitized medium," Journal of Applied Physics, vol. 77, no. 12, pp. 6087-6099, 1995.

[21] S. Torquato, J. D. Beasley, and Y. C. Chiew, "Two-point cluster function for continuum percolation," The Journal of Chemical Physics, vol. 88, no. 10, pp. 6540-6547, 1988.

[22] S. B. Lee and S. Torquato, "Measure of clustering in continuum percolation: computer-simulation of the two-point cluster function," The Journal of Chemical Physics, vol. 91, no. 2, pp. 1173$1178,1989$.

[23] K.-Y. Shin, S.-B. Kim, J.-H. Kim, M. Chung, and P.-S. Jung, "Thermo-physical properties and transient heat transfer of concrete at elevated temperatures," Nuclear Engineering and Design, vol. 212, no. 1-3, pp. 233-241, 2002.
[24] W. Feist, J. Schnieders, V. Dorer, and A. Haas, "Re-inventing air heating: convenient and comfortable within the frame of the Passive House concept," Energy and Buildings, vol. 37, no. 11, pp. 1186-1203, 2005.

[25] S. Grieu, O. Faugeroux, A. Traoré, B. Claudet, and J.-L. Bodnar, "Artificial intelligence tools and inverse methods for estimating the thermal diffusivity of building materials," Energy and Buildings, vol. 43, no. 2-3, pp. 543-554, 2011.

[26] S.-C. Ng and K.-S. Low, "Thermal conductivity of newspaper sandwiched aerated lightweight concrete panel," Energy and Buildings, vol. 42, no. 12, pp. 2452-2456, 2010.

[27] A. Sales, F. R. de Souza, W. N. Dos Santos, A. M. Zimer, and F. D. C. R. Almeida, "Lightweight composite concrete produced with water treatment sludge and sawdust: thermal properties and potential application," Construction and Building Materials, vol. 24, no. 12, pp. 2446-2453, 2010.

[28] E. Underwood, Quantitative Stereology, Addison-Wesley, Reading, Mass, USA, 1970.

[29] B. Lu and S. Torquato, "Lineal-path function for random heterogeneous materials," Physical Review A, vol. 45, no. 2, pp. 922-929, 1992.

[30] H. Singh, A. M. Gokhale, S. I. Lieberman, and S. Tamirisakandala, "Image based computations of lineal path probability distributions for microstructure representation," Materials Science and Engineering A, vol. 474, no. 1-2, pp. 104-111, 2008.

[31] N. Otsu, "A threshold selection method from gray-level histograms," IEEE Transactions on Systems, Man and Cybernetics, vol. 9, no. 1, pp. 62-66, 1979.

[32] MATLAB, Version 7.10 (R2010a), The MathWorks, Natick, Mass, USA, 2010. 

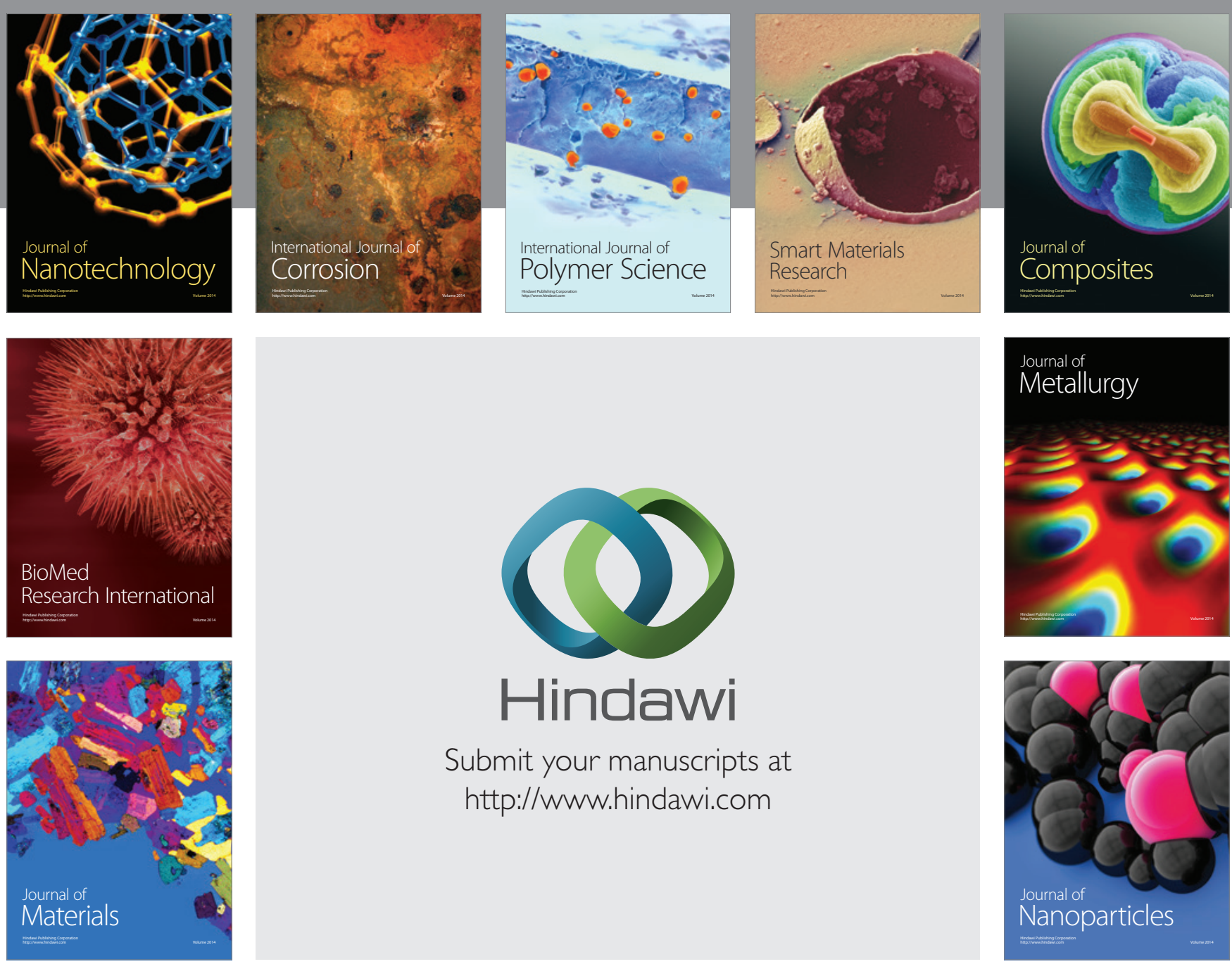

Submit your manuscripts at http://www.hindawi.com
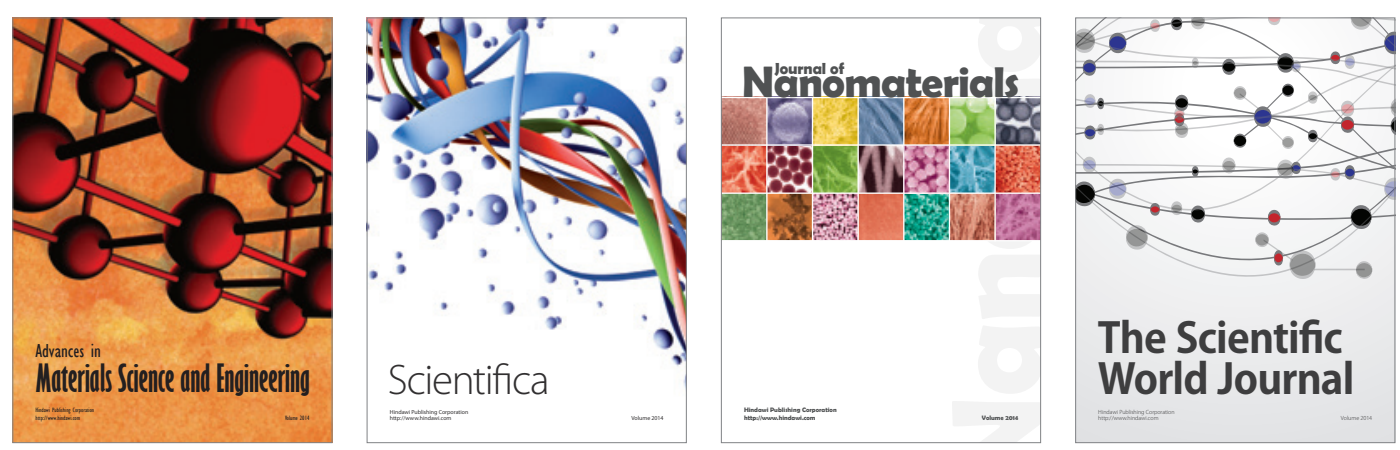

\section{The Scientific World Journal}
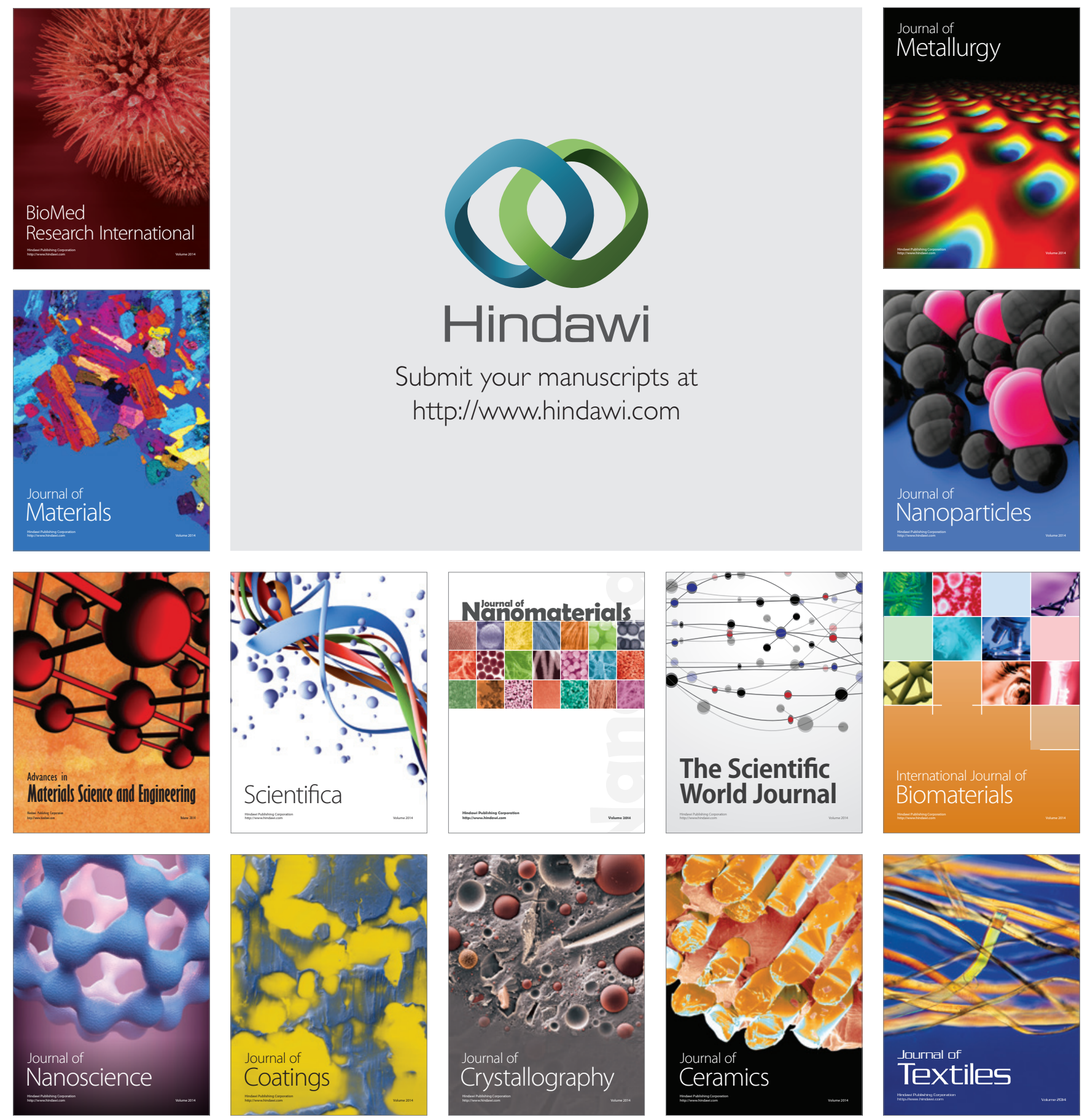